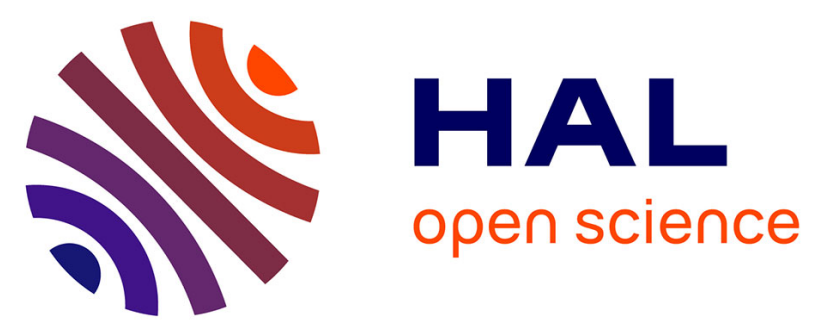

\title{
Application of a response surface method to the optimal design of the wall temperature profiles in extrusion die
} Nadhir Lebaal, Stephan Puissant, Fabrice Schmidt

\section{To cite this version:}

Nadhir Lebaal, Stephan Puissant, Fabrice Schmidt. Application of a response surface method to the optimal design of the wall temperature profiles in extrusion die. International Journal of Material Forming, 2010, 3 (1), pp.47-58. 10.1007/s12289-009-0416-x . hal-01716275

\author{
HAL Id: hal-01716275 \\ https://hal.science/hal-01716275
}

Submitted on 15 Feb 2019

HAL is a multi-disciplinary open access archive for the deposit and dissemination of scientific research documents, whether they are published or not. The documents may come from teaching and research institutions in France or abroad, or from public or private research centers.
L'archive ouverte pluridisciplinaire HAL, est destinée au dépôt et à la diffusion de documents scientifiques de niveau recherche, publiés ou non, émanant des établissements d'enseignement et de recherche français ou étrangers, des laboratoires publics ou privés. 


\title{
Application of a response surface method to the optimal design of the wall temperature profiles in extrusion die
}

\author{
Nadhir Lebaal • Stephan Puissant • Fabrice Schmidt
}

\begin{abstract}
A new approach to the optimal design of the die wall temperature profile in polymer extrusion processes is presented. In this approach, optimization of the design variables is conducted by a Response Surface Method (RSM) and the Sequential Quadratic Programming (SQP) algorithm. Design of experiment (DoE) needed for the construction of the response surface is used to evaluate the objective and the constraint functions on the basis of a finite element method (FEM). Two designs of experiments are used and the performances of the optimization results are compared with respect to efficiency and ability to obtain a global optimum. Typically, for extrusion die design, the objective function states that the average velocity across the die exit is uniform. Constraints are used to limit the pressure drop in the die. For this purpose, we optimize the wall temperature profile of a coat hanger die in a heterogeneous way, (i.e. the wall temperature may not be constant in the entire die). The melt temperature enables us to locally control the viscosity, which influences the flows in the various zones. The effect of the design variables in the objective and constraint functions is investigated using Taguchi method. The flow analysis results are then combined
\end{abstract}

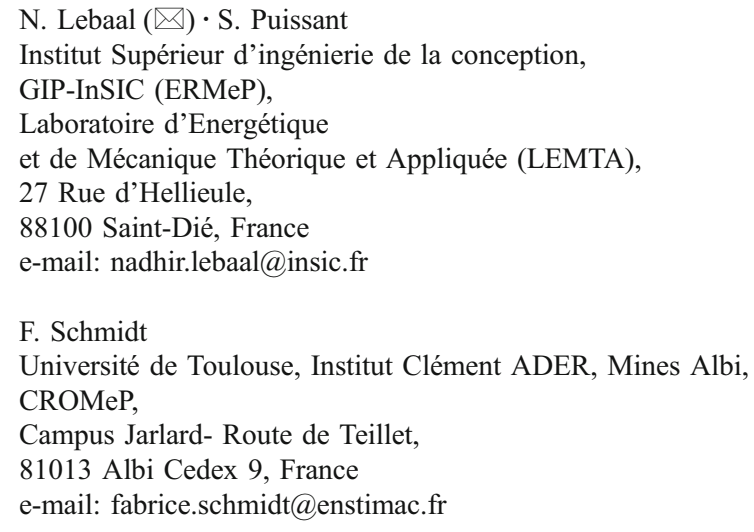

e-mail: fabrice.schmidt@enstimac.fr

with an automatic optimization algorithm to provide a new profile of the die wall temperature distributions.

Keywords Optimization · DoE - Response surface method · FEM

\section{Introduction}

To obtain the optimal parameters during polymer extrusion processes, the numerical simulation imposed itself as an important tool by giving access to difficult orders of magnitudes of physical parameters obtain by the experiment. Moreover it improves the comprehension of the process. Far from being a substitute for the experiment, the numerical simulation allows a time saving and a substantial economy. This is obtained by reducing the number of experiments to be carried out when analyzing the process before its realization. However, obtaining the optimum parameters is often the result of a difficult work of trial and error, during which the various solutions are tested and modified.

The majority of the early works on the extrusion process use the geometrical parameters to optimise the velocity distribution at the die exit [1-4]. The channel geometry of a flat die should be optimized such that a uniform velocity distribution at the die exit is obtained without excessively increasing the pressure drop across the die. Within this framework Smith [5] optimizes a flat die design to well operate at multiple temperatures. The author shows that the exit velocity distribution is influenced by the melt temperature (the material properties for the power law fluid model are varied according to the melt temperature). To simplify the optimisation approach, the lubrication approximations to model isothermal flow of power-law fluids are used. 
Design sensitivities needed for the gradient-based constraint optimization algorithm are evaluated with the adjoint variable method to improve the performance and accuracy of the computations.

If the non isothermal flow is not accounted while optimizing the die [6] the computed velocity pressure fields are expected to have large errors [5]. The non-isothermal flow of a power-law fluid in a coat-hanger die is studied by Vergnes et al. [7]. The authors employed the finite difference method to solve the two dimensional equilibrium and thermal equations (flow field by considering the thermal regulation). The pressure, residence time, and temperature distribution are obtained. The authors show that the thermal regulation and temperature dependence of the viscosity have a large effect on the flow uniformity at the end of the die. Schläfli [8] made an experimental study on the effect of the difference of the temperature between the material and the die on the velocity distribution at the exit of a Coat-hanger melt distributor. He noted that the difference in temperature has a more important effect on the final zone of the distribution channel because of the more important residence time. Within the framework, Vergnes et al. [9] were interested in the manufacture of a tube. The problem encountered during this manufacture is the presence in the section of the tube of a symmetrical extra thickness. This defect is due to the existence, in the entry of the die, of two hotter zones of melt due to the flow in the twin screw extrusion machine. These hot zones separate to flow out on each side of the punch, providing, because of their lower viscosity, more important local flows and thus, extra thicknesses at the exit. To obtain a homogeneous distribution of the flow at the die exit, instead of imposing a homogeneous temperature of regulation, they imposed a heterogeneous temperature of regulation compared to the cold and hot zones. The results show that with this heterogeneous wall temperature, they obtained a practically homogeneous distribution of thickness all around the die. This possibility of heterogeneous thermal regulation was applied and exists at the industrial level. Fradette et al. [10] optimize the cooling channels diameter and position for the cooling layout of vacuum calibrators used in the extrusion of PVC profiles. The required objective is to minimize the average temperature to decrease the time of cooling and to standardize the temperatures to improve quality. The methodology is based on the finite element resolution of the heat transfer conduction problem in the calibrator and the profile coupled to the Fletcher-Reeves optimization algorithm.

For complex geometries, the computational resources and time required for analysis of extrusion dies are considerable. Consequently, the use of evolution strategy algorithm to optimize the extrusion die in three-dimensional analysis is less attractive to designers because of the unacceptable turnaround time for results. The purpose of this work is to deal with this inconvenient disadvantage, by using an automatic optimisation algorithm and several strategies which permit to avoid the local minima and by this fact without increasing the computing time which becomes penalizing for three-dimensional simulations. The non isothermal flow analysis results are then combined with an automatic optimisation algorithm that is based on a response surface methodology [11] and a non linear constraint algorithm SQP to optimize the wall temperature profile of a coat hanger die in a heterogeneous way.

\section{Mesh size sensitive}

In this section we examine and analyze the effect of the mesh size over CPU time and on some results: pressure in the die entrance, and the exit velocity distribution. The mesh distribution was improved by dividing the die into four zones. The first zone represents the entry of the die and the manifold. The other zones $(2,3,4)$ represent the zones of low thickness of the die (land), which are respectively: the triangle, the relaxation zone, and the lips, as Fig. 1 indicates. On these zones we will impose various sizes of finite elements. Considering the great shape ratio (thickness over width) (Fig. 2) and to respect some number of node on the thickness the anisotropic mesh is privileged (i.e. size of element which varies according to each direction). Seven mesh sizes were analyzed. In Table 1, we present the characteristics of each mesh: elements size according to each direction and for the various zones, node and element numbers, CPU time and pressure drop in the die.

We compare the pressure obtained by the various mesh size with that obtained by the finest mesh taken as reference. Thus, we note, according to the results obtained (Table 1), that by refining the mesh and by using an adapted mesh for each zone, the difference between the pressures becomes negligible; on the other hand, the CPU time becomes more important.

In order to quantify the influence of the mesh density on the exit velocities distribution, we represent in Fig. 2 the results corresponding to the various mesh size.

We note that the exit velocities distribution converge by refining the mesh with very close values. By analyzing more closely the curves, we remark that a stabilization of the results is established starting from mesh 4 .

\section{Optimisation procedure}

Optimization variables

In order to keep the same geometry and obtain a homogeneous velocity distribution at the die exit, an 


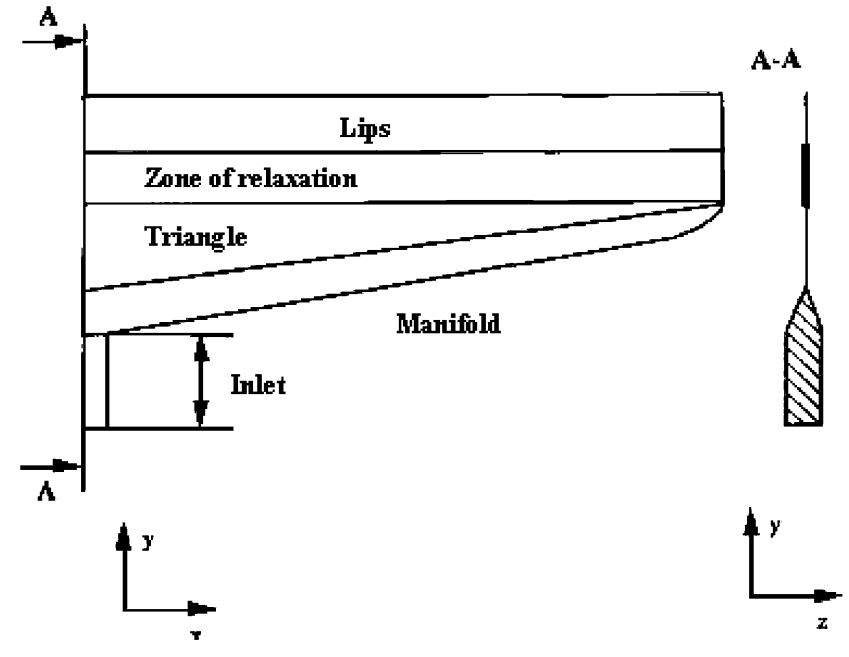

Fig. 1 Coat hangers die geometry

optimization problem is formulated, which consists in optimizing the temperature of regulation of the flat die. For this procedure, the geometry of the die as well as the mesh remains constant. On the other hand, physical parameters, which represent the wall die temperatures of regulation, will be used as optimization variables to obtain a homogeneous exit velocity distribution. 6 temperatures of regulation "T1, T2, T3, Ta, Tb and $\mathrm{Tc}$ " are imposed according to the width of the die (Fig. 3). Because of symmetry, we represented only half die. To decrease the number of optimization variables, we use a control curve obtained by Kriging, to allow the control of the die wall temperature profile, and to decrease the number of variables to 3 .
Among the 6 optimization variables $\mathrm{Ta}, \mathrm{Tb}$ and $\mathrm{Tc}$ are active; whereas, T1, T2, and T3 are passive. This means that $\mathrm{T} 1$ varies like $\mathrm{Ta}$, at the distance of the width of the die from 0 to $200 \mathrm{~mm}$. From the three other temperatures (active variables), we can plot a curve using the Kriging interpolation to represent other die wall regulation temperatures (passive variables).

The optimization problem is thus resolved with three parameters ( $\mathrm{Ta}, \mathrm{Tb}$, and $\mathrm{Tc}$ ). We can also add to it the applied constraint conditions so that the temperatures do not exceed an imposed value. Initially, the temperature of regulation is constant through all the width of the die $\mathrm{T}=230^{\circ} \mathrm{C}$. This constant temperature gives a non uniform velocity distribution. Velocities are more important of about $50 \%$ at the edge and weaker of about $25 \%$ in the middle of the die compared to the average velocity. During the optimisation processes the variables permit the variation between $200^{\circ} \mathrm{C}$ and $280^{\circ} \mathrm{C}$. The maximum temperature of $280^{\circ} \mathrm{C}$ is chosen i.e. there is no polymer degradation.

\section{Objective and constraint function}

The optimisation problem consists to obtain the optimal wall temperatures profile of coat hanger die so that the objective function based on the exit velocity distribution is minimal; the sheet thickness will be then homogeneous. This represents a very important material profit considering the quantity of the extruded polymer sheets each year. The objective function $(f(x))$ represents the total relative variation on the exit velocity distribution. In order to ensure that the pressure at the entry of the die $\left(P^{\mathrm{e}}\right)$ does not increase compared to the pressure obtained by the initial wall temperature $\left(P_{0}^{e}\right)$, a constraint function $(g(x))$ based on the
Fig. 2 Mesh size effects on the exit velocity distribution

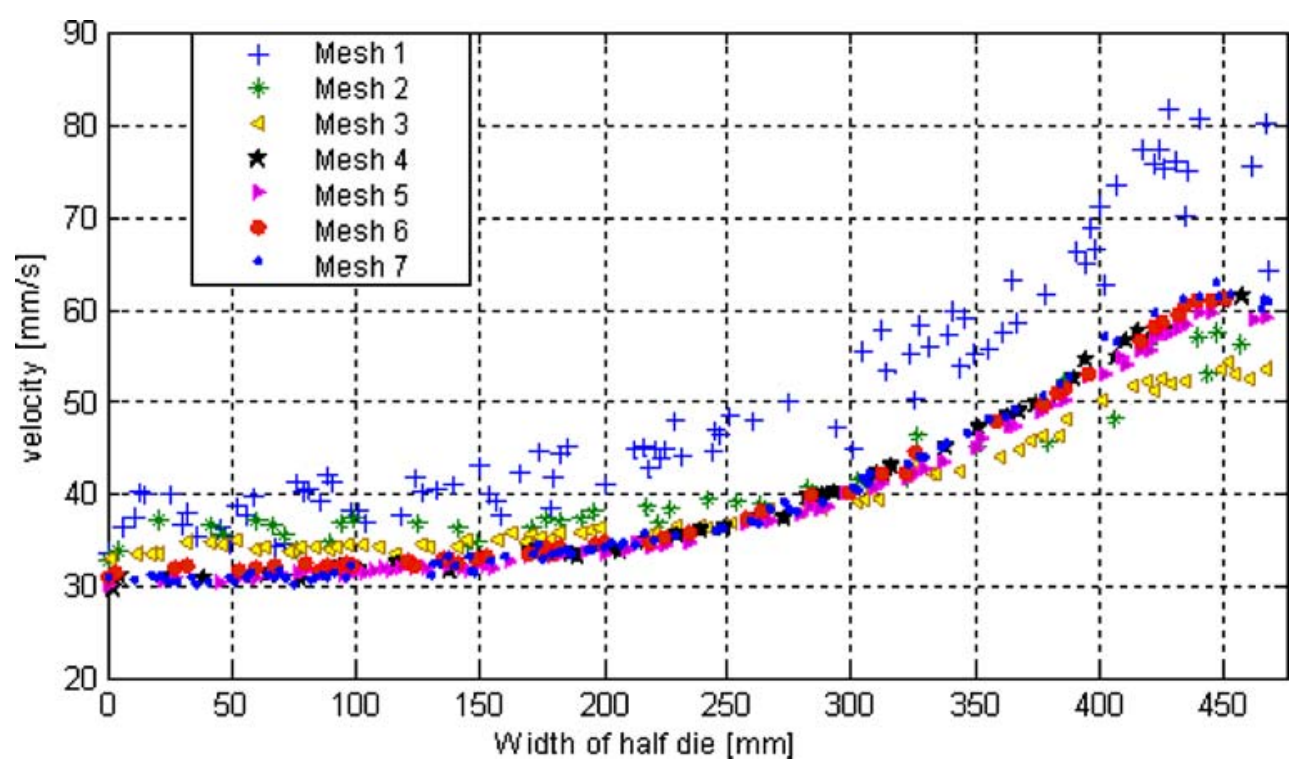


Table 1 Characteristics of the various mesh size

\begin{tabular}{|c|c|c|c|c|c|c|c|c|c|c|c|c|c|c|c|c|}
\hline \multirow[t]{3}{*}{ Mesh } & \multicolumn{12}{|c|}{$\begin{array}{l}\text { Mesh size }[\mathrm{mm}] \text { according to each direction for the various } \\
\text { zones }\end{array}$} & \multirow[t]{3}{*}{ Nodes } & \multirow[t]{3}{*}{ Elements } & \multirow[t]{3}{*}{ Pressure $[\mathrm{MPa}]$} & \multirow[t]{3}{*}{ CPU Time [min] } \\
\hline & \multicolumn{3}{|c|}{ Zone 1} & \multicolumn{3}{|c|}{ Zone 2} & \multicolumn{3}{|c|}{ Zone 3} & \multicolumn{3}{|c|}{ Zone 4} & & & & \\
\hline & $\mathrm{x}$ & $\mathrm{y}$ & $\mathrm{z}$ & $\mathrm{x}$ & $\mathrm{y}$ & $\mathrm{z}$ & $\mathrm{x}$ & $\mathrm{y}$ & $\mathrm{z}$ & $\mathrm{x}$ & $\mathrm{y}$ & $\mathrm{z}$ & & & & \\
\hline (1) & 4 & 4 & 4 & 2 & 2 & 2 & 2 & 2 & 2 & 1 & 1 & 1 & 135357 & 659761 & 4.87 & 267 \\
\hline (2) & 6 & 3 & 7 & 6 & 1.5 & 6 & 6 & 1.5 & 6 & 5 & 1 & 5 & 20708 & 94113 & 3.95 & 14 \\
\hline (3) & 6 & 4 & 6 & 6 & 1 & 6 & 6 & 1.5 & 6 & 3 & 0.75 & 3 & 24380 & 112209 & 3.98 & 26 \\
\hline (4) & 6 & 4 & 6 & 4 & 0.75 & 4 & 5 & 1 & 5 & 3 & 0.5 & 3 & 35837 & 176806 & 4.44 & 49 \\
\hline (5) & 5 & 2 & 5 & 4 & 0.75 & 4 & 4 & 1 & 4 & 3 & 0.5 & 3 & 60356 & 302588 & 4.43 & 85 \\
\hline (6) & 4 & 1 & 4 & 2 & 0.5 & 2 & 2 & 0.5 & 2 & 2 & 0.37 & 2 & 104208 & 532018 & 4.48 & 192 \\
\hline Reference (7) & 2 & 1 & 2 & 1 & 0.37 & 1 & 1 & 0.37 & 1 & 1 & 0.37 & 1 & 220888 & 1146689 & 4.46 & 431 \\
\hline
\end{tabular}

pressure at the entry of the die is used. This optimization problem is represented by the Eq. (1).

$$
\left\{\begin{array}{l}
\min f(x)=\frac{E}{E_{0}} \\
g(x)=\frac{P^{e}-P_{0}^{e}}{P_{0}^{e}} \leq 0 . \\
x_{\min } \leq x \leq x_{\max }
\end{array}\right.
$$

The velocity dispersion (E), is defined as:

$$
E=\left(\frac{1}{N} \sum_{i=1}^{N}\left(\frac{\left|v_{i}-\bar{v}\right|}{\bar{v}}\right)\right)
$$

where $E_{0}$ and $P_{0}$ are respectively the total relative variation of the exit velocity distribution and the pressure in the initial die, $\mathrm{N}$ is the total number of nodes at the die exit in the middle plane; $\nu_{\mathrm{i}}$ is the velocity at an exit node, and $\bar{v}$ is the average exit velocity which is defined as follows:

$\bar{v}=\frac{1}{N} \sum_{i=1}^{N} v_{i}$

$x_{\min }$ and $x_{\max }$ are the lower and higher limits of optimization variables, with:

$$
x_{\min }=\left\{\begin{array}{l}
T a_{\min }=200^{\circ} \mathrm{C} \\
T b_{\min }=200^{\circ} \mathrm{C} \\
T c_{\min }=200^{\circ} \mathrm{C}
\end{array}\right\} \text { et } x_{\max }=\left\{\begin{array}{l}
T a_{\max }=280^{\circ} \mathrm{C} \\
T b_{\max }=280^{\circ} \mathrm{C} \\
T c_{\max }=280^{\circ} \mathrm{C}
\end{array}\right\}
$$

Fig. 3 Wall temperatures distribution according to the width of the die (optimization variables)

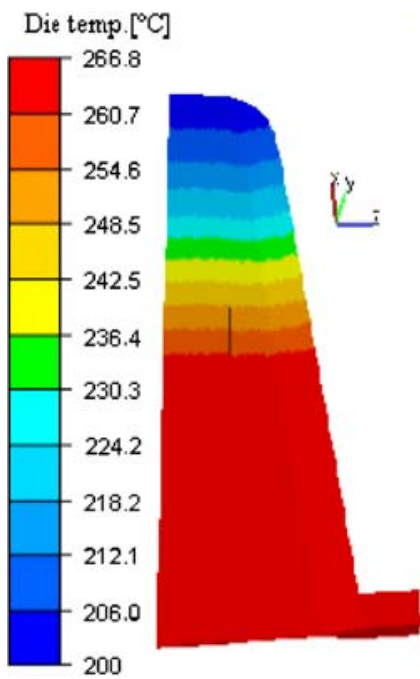


Table 2 Response of the objective and constraint functions using CCD

\begin{tabular}{clllrrc}
\hline Responses & $\mathrm{T}_{\mathrm{a}}$ & $\mathrm{T}_{\mathrm{b}}$ & $\mathrm{T}_{\mathrm{c}}$ & $E$ en $\%$ & $\mathrm{~g}$ & Pressure [bar] \\
\hline 1 & $2(-1 / \alpha)$ & $2(-1 / \alpha)$ & $2(-1 / \alpha)$ & 23.4 & 0.1978 & 5.33 \\
2 & $4(+1 / \alpha)$ & $2(-1 / \alpha)$ & $2(-1 / \alpha)$ & 7.04 & -0.189 & 4.36 \\
3 & $2(-1 / \alpha)$ & $4(+1 / \alpha)$ & $2(-1 / \alpha)$ & 30.84 & 0.1109 & 4.94 \\
4 & $4(+1 / \alpha)$ & $4(+1 / \alpha)$ & $2(-1 / \alpha)$ & 9.95 & -0.0859 & 4.06 \\
5 & $2(-1 / \alpha)$ & $2(-1 / \alpha)$ & $4(+1 / \alpha)$ & 27.52 & 0.1758 & 5.23 \\
6 & $4(+1 / \alpha)$ & $2(-1 / \alpha)$ & $4(+1 / \alpha)$ & 11.92 & -0.0374 & 4.28 \\
7 & $2(-1 / \alpha)$ & $4(+1 / \alpha)$ & $4(+1 / \alpha)$ & 32.51 & 0.0937 & 4.86 \\
8 & $4(+1 / \alpha)$ & $4(+1 / \alpha)$ & $4(+1 / \alpha)$ & 12.51 & -0.1 & 4.00 \\
9 & $3(0)$ & $3(0)$ & $3(0)$ & 16.57 & 0.0361 & 4.61 \\
10 & $1(-1)$ & $3(0)$ & $3(0)$ & 51.32 & 0.3503 & 6.01 \\
11 & $5(+1)$ & $3(0)$ & $3(0)$ & 8.14 & -0.1804 & 3.64 \\
12 & $3(0)$ & $1(-1)$ & $3(0)$ & 16.98 & 0.1407 & 5.07 \\
13 & $3(0)$ & $5(+1)$ & $3(0)$ & 25.40 & -0.0490 & 4.23 \\
14 & $3(0)$ & $3(0)$ & $1(-1)$ & 13.70 & 0.0558 & 4.70 \\
15 & $3(0)$ & $3(0)$ & $5(+1)$ & 21.75 & 0.0111 & 4.50 \\
\hline
\end{tabular}

The maximum temperature of $280^{\circ} \mathrm{C}$ is chosen so there is no polymer degradation.

\section{Sensitive analysis}

The central composite design (CCD) is the most popular class of designs used for fitting a second-order model [11, 12]. The rotatability is important for the second-ordermodel to provide good predictions throughout the region of interest. Because the purpose of this work is the optimization and the location of the optimum parameters, is important to use a design that provides equal precision of estimation in all directions. For this, the CCD is made rotatable.

In order to evaluate the effects of the optimization variables on the objective and constraint functions, design of experiments method was used to estimate the effects of the die wall temperature profile on the pressure drop and on the exit velocities distribution. In this study, three parameters were considered with five levels each. According to the CCD, 15 complete simulations were chosen. The various levels are represented from 1 to 5 respectively giving the values of the minimum to the maximum of each variable. For this, we have calculated the effect of each variable over all the region of interest by studying the contribution of each variable on the response variation "objective and constraint function (Table 2).

Figure 4 represent the average effect of the optimization variables on the objective function (velocities distribution at the die exit). It is noted that the temperature Ta has a most important effect on the velocities distribution. This temperature is located at the die middle, where the velocities are lowest. For the other temperatures, $\mathrm{Tb}$ and $\mathrm{Tc}$ seem having less important effects. We notice that the combination which offers the minimum of the objective function corresponds to the maximum of the temperature Ta, and the minimum of the
Fig. 4 Average effect of variables levels on the objective function (exit velocity distribution)

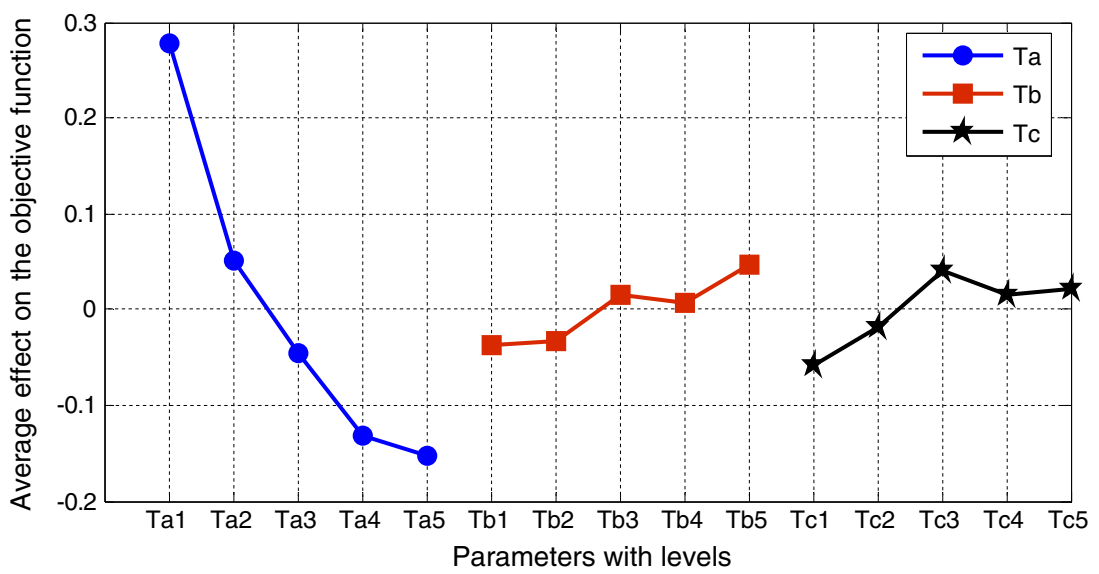


Fig. 5 Average effect of variables levels on the constraint function (pressure)

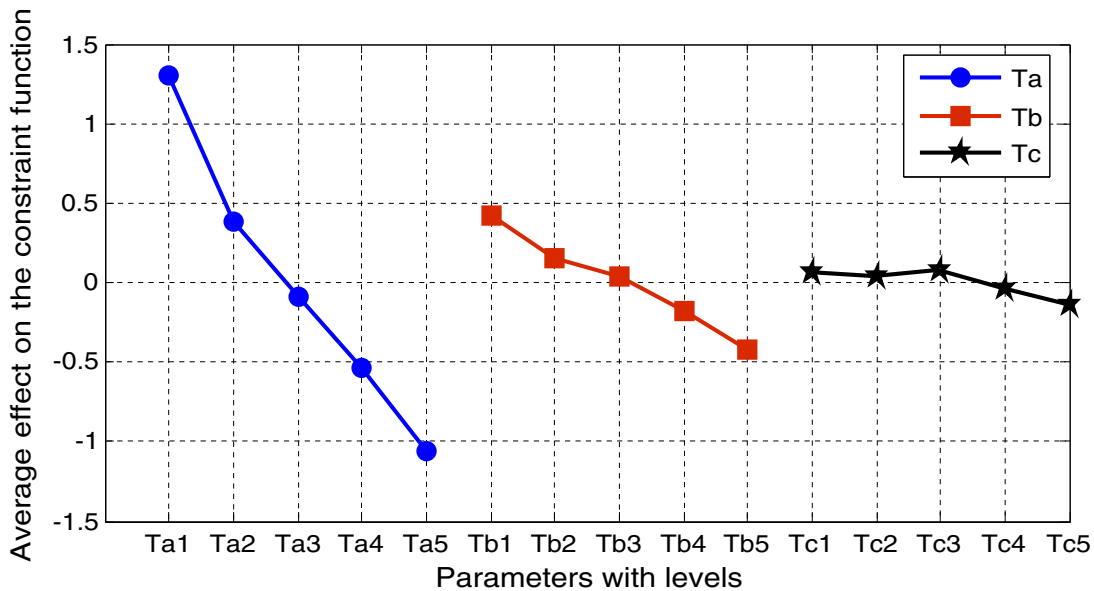

temperatures $\mathrm{Tb}$, and $\mathrm{Tc}$. This allows changing the polymer viscosity and controlling the flow locally. At this place, or the air-gap is weaker, that compensates for the lack of flow on this zone. On the other hand, for the zones located on the edge of the die (more important velocities), the temperatures of regulation $\mathrm{Tb}$ and $\mathrm{Tc}$, which are low, generate an increase in viscosity, and thus a reduction in the flow.

Concerning their effects on the pressure (Fig. 5) we can classify them by descending order. The greatest effect on the pressure variation is given by $\mathrm{Ta}$, then $\mathrm{Tb}$ and finally $\mathrm{Tc}$ which has the weakest effect. The combination, of the optimization variables, which offers the minimum of the constraint function (pressure), corresponds to the maximum of the temperatures of regulation. That makes it possible to increase the melt temperature. Into consequent, the melt viscosity decreases, which favourite the flow with less pressure loss.

Response surface method

The objective and constraint functions are implicit, compared to the optimization parameters and their evaluation requires a large amount of computing time using three dimensional analyses. In order to decrease the evaluation number of the objective and constraint functions we use in this work the response surface method $[11,13]$. This method consists in the construction of an approximate expression of the objective and constraint functions starting from a limited number of evaluations of the real functions. In order to obtain a good approximation, we used a Kriging interpolation described in next section. In this method, the approximation is computed by using the evaluation points obtained using Design of Experiments technique.

Two optimization results are built (conducted) using two DoE for the construction of the R.S (Fig. 6) the firs one is the Enriched Composite Design (ECD) and the second is the Central Composite Design (CCD).

Kriging interpolation

Kriging is an interpolation technique [14, 15] which allows to model very efficiently complex functions. It is applied here to represent the response surface in an explicit form according to the variables of optimization. The explicit

Fig. 6 Design of experiment

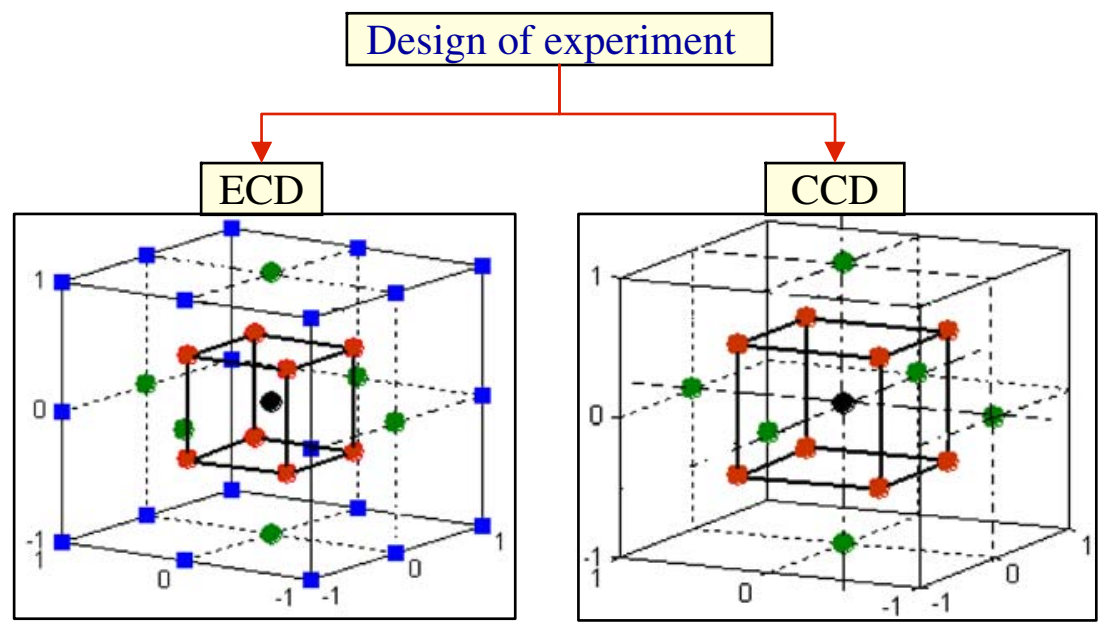


Fig. 7 The flowchart of the adopted optimization strategy

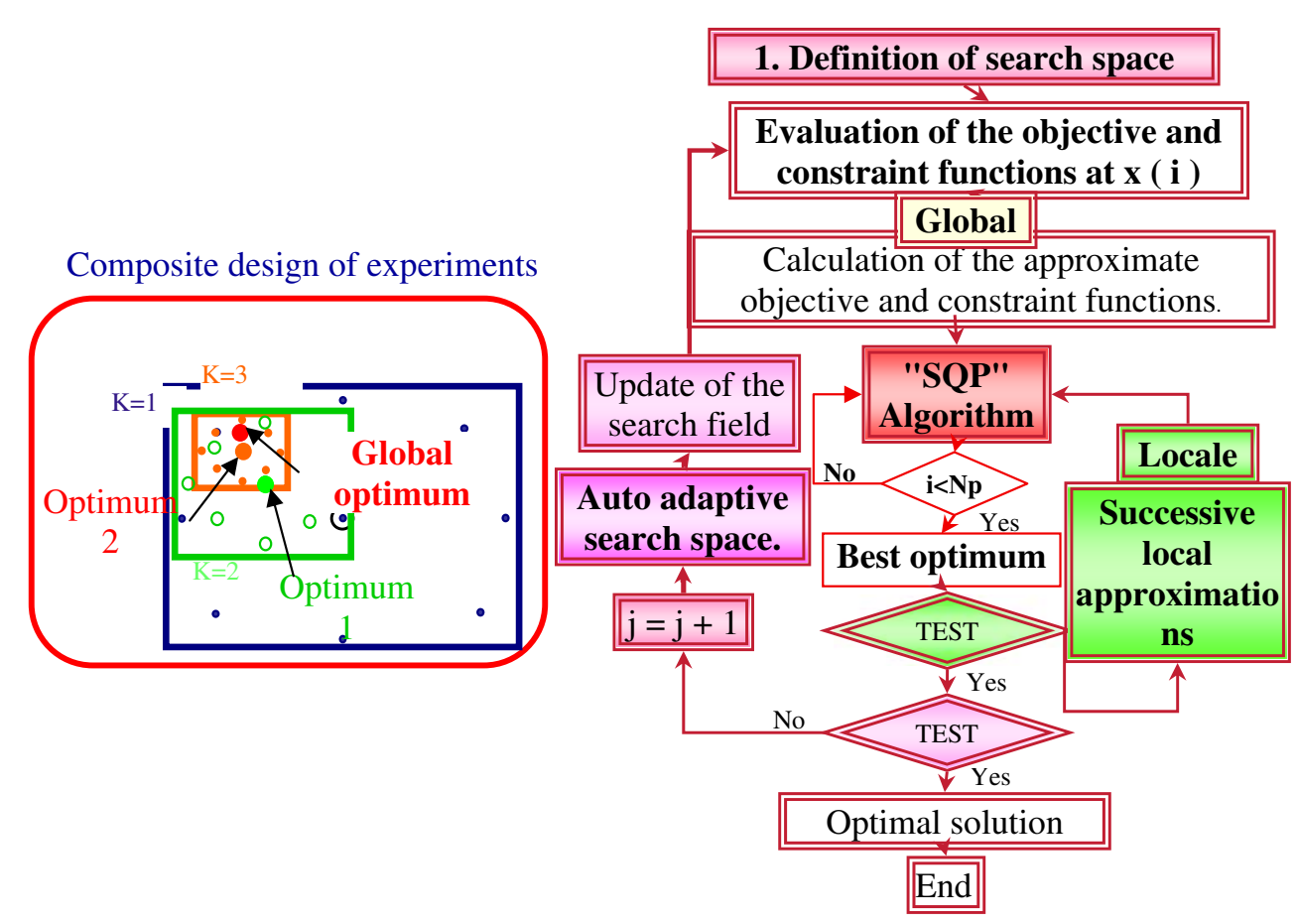

relationship of the objective and constraint function can be expressed as follows:

$\widetilde{J}(x)=p^{T}(x) a+Z(x)$

with, $p(x)=\left[p_{1}(x), \ldots, p_{m}(x)\right]^{T}$, where $m$ denotes the number of the basis function in regression model, $\alpha=$ $\left[\alpha_{1}, \ldots, \alpha_{m}\right]^{T}$ is the coefficient vector the, $x$ is the design variables, $\widetilde{J}(x)$ is the unknown objective or constraint interpolate function, and $Z(x)$ is the random fluctuation. The term $p^{\mathrm{T}}(x) a$ in Eq. (5) indicates a global model of the design space, which is similar to the polynomial model in a
Moving Least Squares (MLS) approximation. The second part in Eq. (5) is a correction of the global model. It is used to model the deviation from $p^{\mathrm{T}}(x) a$ so that the whole model interpolates response data from the function.

The output responses from the function are given as:

$F(x)=\left\{f_{1}(x), f_{2}(x), \ldots f_{n}(x)\right\}$

From these outputs the unknown parameters $\alpha$ can be estimated:

$\alpha=\left(P^{T} R^{-1} P\right)^{-1} P^{T} R^{-1} F$
Table 3 Summary of the results for the two optimization cases

\begin{tabular}{|c|c|c|c|c|c|c|c|}
\hline \multirow{3}{*}{$\begin{array}{l}\text { DoE } \\
\text { CPU Time } \\
\text { Iterations }\end{array}$} & \multirow{3}{*}{$\begin{array}{l}\text { Initial } \\
0\end{array}$} & \multirow{2}{*}{\multicolumn{3}{|c|}{$\frac{\text { Case } 1 E C D}{5 \text { day and } 9 \mathrm{~h}}$}} & \multirow{2}{*}{\multicolumn{3}{|c|}{$\frac{\text { Case } 2 \mathrm{CCD}}{2 \text { day and } 9 \mathrm{~h}}$}} \\
\hline & & & & & & & \\
\hline & & 1 & 2 & 3 & 1 & 2 & 3 \\
\hline Objective function & 1 & 0.209 & 0.083 & 0.064 & 1.13 & 0.149 & 0.076 \\
\hline velocity dispersion $\mathrm{E}$ & $21 \%$ & $4.35 \%$ & $1.73 \%$ & $1.33 \%$ & $23.5 \%$ & $3.1 \%$ & $1.58 \%$ \\
\hline Improvement in velocities distribution & - & - & - & $93.6 \%$ & - & - & $92.4 \%$ \\
\hline constraint function $\left(P^{e} / P_{0}^{e}\right)$ & 1 & 0.68 & 0.71 & 0.73 & 0.83 & 0.89 & 0.84 \\
\hline Improvement in pressure drop & - & - & - & $27 \%$ & - & - & $16 \%$ \\
\hline Pressure[MPa] & 4.426 & 3.032 & 3.158 & 3.223 & 3.678 & 3.947 & 3.742 \\
\hline Temperature $\mathrm{T}_{1}\left[{ }^{\circ} \mathrm{C}\right]$ & 230 & 280 & 280 & 276 & 280 & 256 & 260.5 \\
\hline Temperature Ta $\left[{ }^{\circ} \mathrm{C}\right]$ & 230 & 280 & 280 & 276 & 280 & 256 & 260.5 \\
\hline Temperature $\mathrm{T}_{2}\left[{ }^{\circ} \mathrm{C}\right]$ & 230 & - & - & 266 & - & - & 246 \\
\hline Temperature $\mathrm{Tb}\left[{ }^{\circ} \mathrm{C}\right]$ & 230 & 274 & 257 & 257 & 200 & 224 & 235 \\
\hline Temperature $\mathrm{T}_{3}\left[{ }^{\circ} \mathrm{C}\right]$ & 230 & - & - & 244.5 & - & - & 222 \\
\hline Temperature $\mathrm{Tc}\left[{ }^{\circ} \mathrm{C}\right]$ & 230 & 239.5 & 227.5 & 224 & 200 & 200 & 200 \\
\hline
\end{tabular}




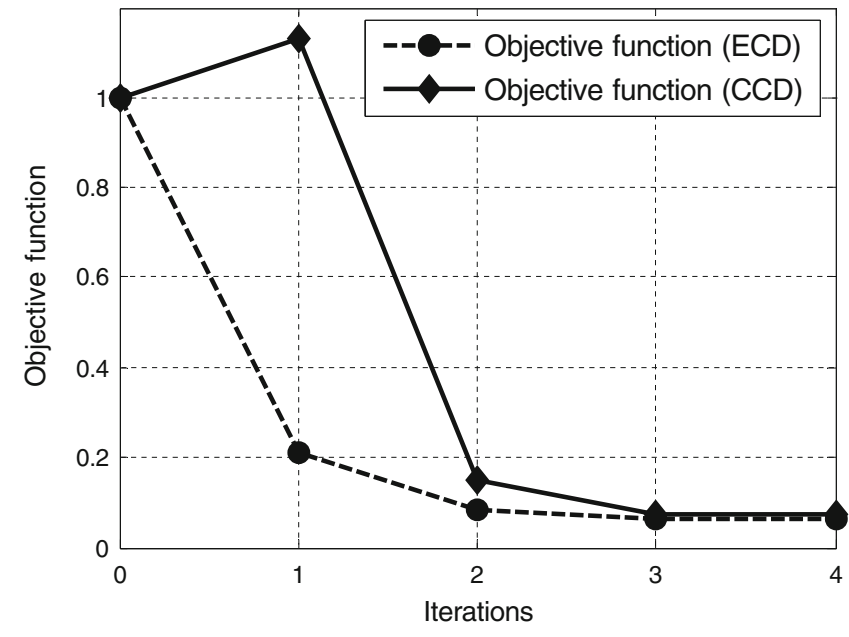

Fig. 8 Convergence history during an optimization process on the objective functions

Where $P$ is a vector including the value of $p(x)$ evaluated at each of the design variables and $R$ is the correlation matrix, which is composed of the correlation function evaluated at each possible combination of the points of design:

$$
\begin{aligned}
& R=\left[\begin{array}{ccc}
R\left(x_{1}, \mathrm{x}_{1}\right) & \cdots & R\left(x_{1}, \mathrm{x}_{n}\right) \\
\vdots & \ddots & \vdots \\
R\left(x_{n}, \mathrm{x}_{1}\right) & \cdots & R\left(x_{n}, \mathrm{x}_{n}\right)
\end{array}\right] \\
& +\left[\begin{array}{cccc}
w\left(x-x_{1}\right) & 0 & \cdots & 0 \\
0 & w\left(x-x_{2}\right) & \cdots & 0 \\
\vdots & \vdots & \vdots & \vdots \\
0 & 0 & \cdots & w\left(x-x_{n}\right)
\end{array}\right]
\end{aligned}
$$

$R_{i j}=\left(\left|x_{i}-x_{j}\right|\right)^{3}$
A weight function of Gaussian type with a circular support is adopted for the Kriging interpolation because its derivatives with respect to the coordinates exist to any desired order. It takes the form

$W_{i}(x)=\left\{\begin{array}{cl}\left(1-\frac{\exp \left(-\left(d_{i} / c\right)^{2}\right)-\exp \left(-\left(r_{w} / c\right)^{2}\right)}{1-\exp \left(-\left(r_{w} / c\right)^{2}\right)}\right) * \chi & d_{i} \leq r_{w} \\ \chi & d_{i} \geq r_{w}\end{array}\right.$

where $d_{i}=\sqrt{\sum_{J=1}^{n}\left(x^{J}-x_{(i)}^{J}\right)^{2}}$ is the distance from a discrete node $x_{\mathrm{i}}$ to a sampling point $\mathrm{x}$ in the domain of support with radius $r_{\mathrm{w}}$, and $\mathrm{c}$ is the dilation parameter. $c=$ $\frac{r_{w}}{4}$ is used in computation. $0 \leq \chi \leq 1$ represents the degree of the importance of the weight function.

The second part in Eq. (5) is in fact an interpolation of the residuals of the regression model $p^{T}(x) \alpha$. Thus, all response data will be exactly predicted; is given as:

$Z(x)=r^{T}(x) \beta$

Where $r^{T}(x)=\left\{R\left(x, x_{1}\right), \cdots, R\left(x, x_{n}\right)\right\}$ The parameters $\beta$ are defined as fallow:

$\beta=R^{-1}(F-P \alpha)$

Optimization strategy

In order to obtain a true global optimum (defined by the exact response) and to respect the non linear constraint function, an efficient optimization strategy using response surfaces are build:

In the first step we use the global response surface, using the DoE defined on the whole research space. The objective and constraint functions will be computed into a limited evaluation response, and we minimize it using a SQP
Fig. 9 Relative variation of the exit velocities distribution with initial and optimal wall die temperature distribution using (ECD \& CCD)

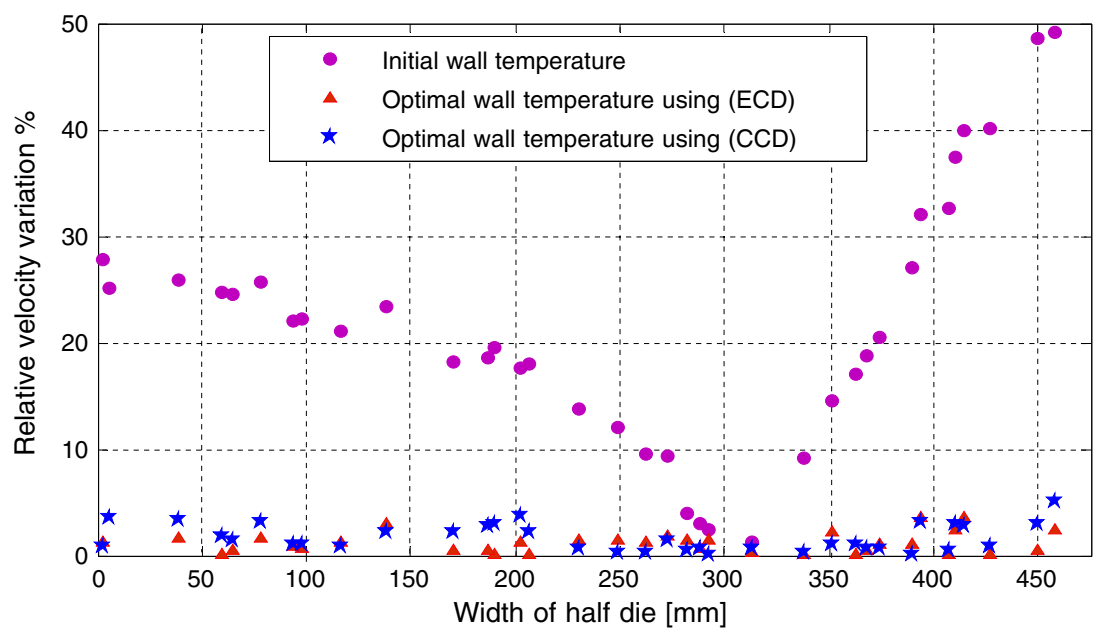


(sequential quadratic programming method). To avoid local minima, and by this fact without increasing the computing time, we change the initial points of the SQP method by all the points of the DoE because the evaluation of the explicit functions does not make problems and does not take much time. When we have obtained the best minimum; successive local approximate problems based on the weight function of Gaussian type are then resolved using an SQP algorithm. This weight function gives, also more importance to the responses which are closer to the minimum and less importance to the other responses, which allows makes the response surface more accurate locally.

During the progression of the optimization procedure (Fig. 7), the region of interest moves and zooms on each optimum by reducing the search space by $1 / 3$. In addition we enriches then the collection $\left\{x^{\mathrm{k}}\right\}$ with the points by the vicinity $\Re_{\text {min }}^{x^{k}}$ giving a new collect $\left\{x^{k+1}\right\}=\left\{x^{k}\right\} \cup \Re_{\text {min }}^{k}$ at the stage $k+1$. At this iteration $(k+1)$ we construct a new approximation $\widetilde{f}^{k+1}$ starting from $\left\{x^{\mathrm{k}+1}\right\}$. The number of evaluation points considered increases because of enriching the collection $\left\{x^{\mathrm{k}}\right\}$ at the vicinity. This carries out to an auto adaptive search space and increases the accuracy when we approach the optimum. These steps are repeated till the new profile temperature satisfies the objective and the constraints function and the iterative procedure stops when the successive points are superposed with a tolerance of $\varepsilon=10^{-3}$.

\section{Numerical application}

The optimization strategy described previously is applied hear based on the response surfaces method and the Kriging
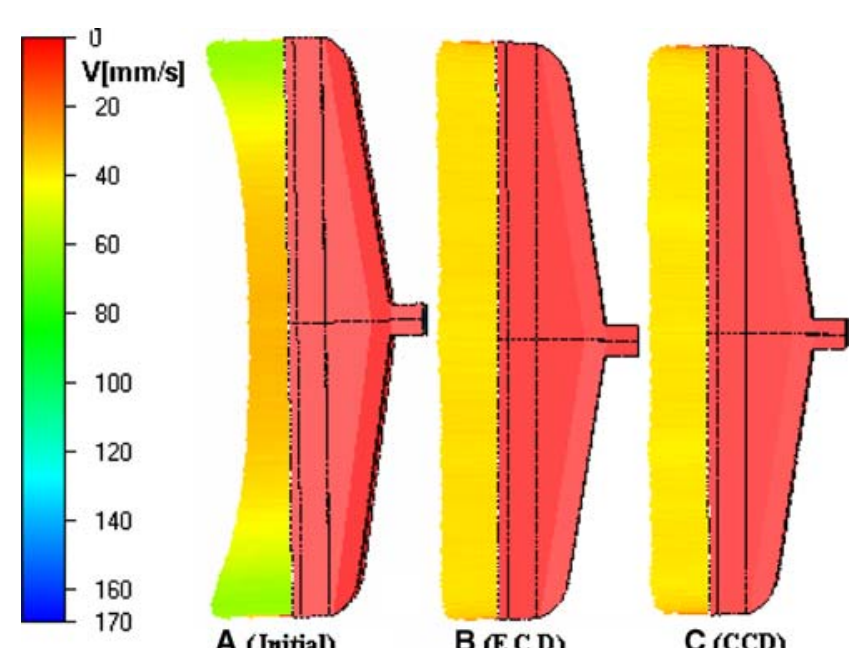

Fig. 10 Exit velocity distributions in the initial (a), and optimized (b, c) temperature of regulation

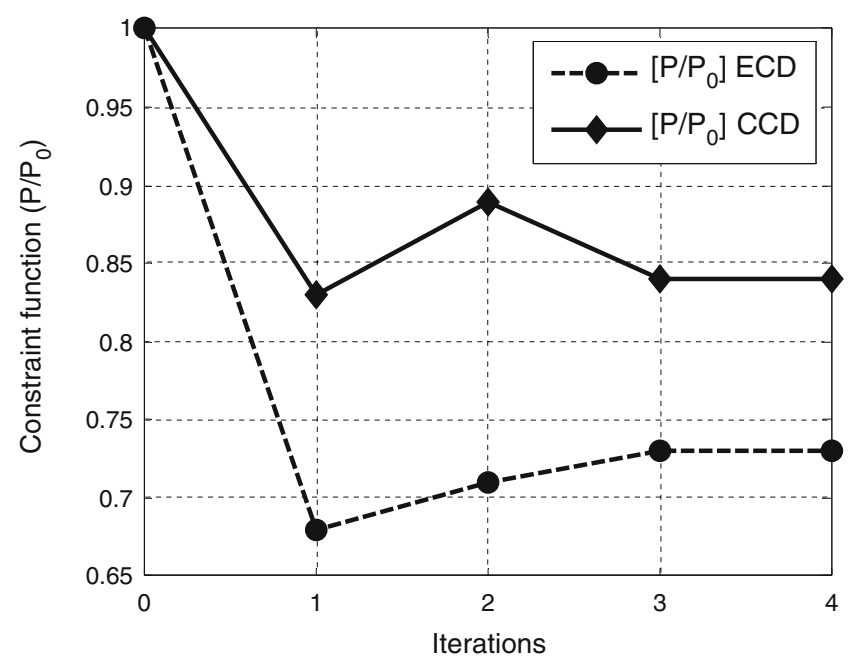

Fig. 11 Convergence history during an optimization process on the constraint functions

interpolation. Two results are presented by using two different design of experiment:

- Case 1: Optimization is carried out by using an Enriched Composite Design of experiment (ECD).

- Case 2: We a Central Composite Design of experiment (CCD).

The process of optimization is carried out and the optimum is obtained from the 3rd iteration; therefore a very fast convergence is obtained for the two results. These calculations take a computing CPU time of 5 days and $9 \mathrm{~h}$ for the ECDs and 2 days and $9 \mathrm{~h}$ for the CCD on a machine Pentium IV, 3 GHz, 1 Go RAM.

The results for the objective function (velocities distribution at the die exit) and the constraint function (pressure)

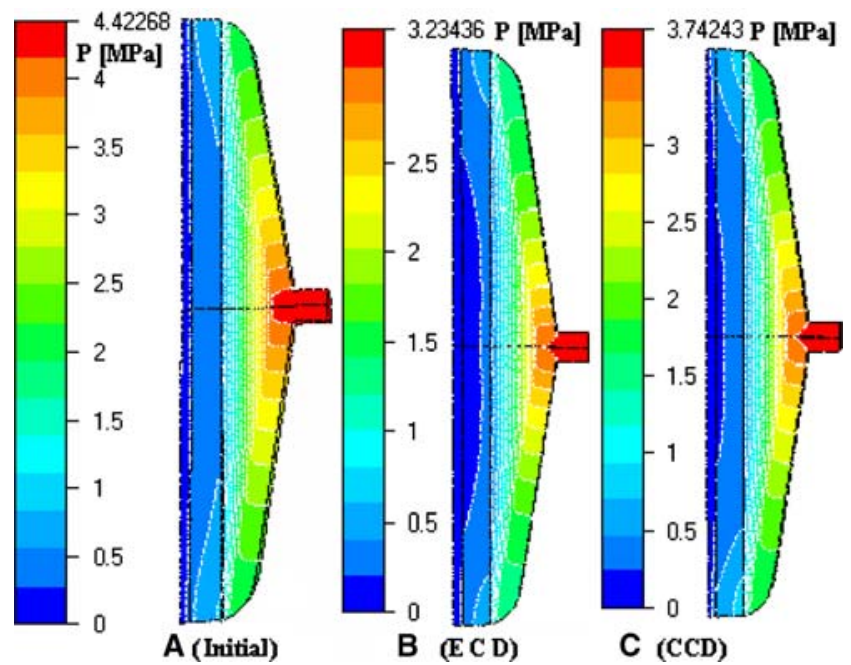

Fig. 12 Pressure distributions in the initial (a), and optimized $(\mathbf{b}, \mathbf{c})$ temperature of regulation 
Fig. 13 Convergence history during an optimization process on the optimizations variables
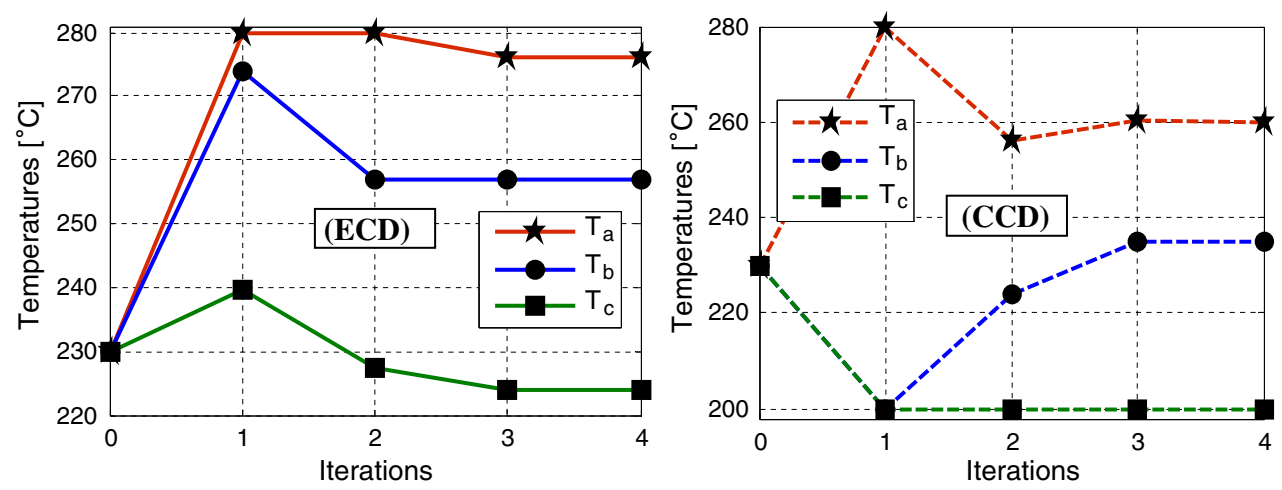

are normalised compared to the results obtained with the initial regulation temperature $\left(\mathrm{T}_{\mathrm{w}}=230^{\circ} \mathrm{C}\right)$. Table 3 shows that with the two results, we find very satisfactory solutions, and that the value of the cost function is reduced moreover than $92 \%$. The constraints on the pressure are respected for the two solutions, as well as the velocities distribution is better. The optimal solution is obtained after three iterations for the two results. On the other hand, the computing time is more important by using the enriched composite design (ECD) (case1). With the use of the CCD (case2), we can obtain a reduction of CPU time of about $66 \%$. The optimum parameters obtained are given in Table 3.

The Fig. 8 represents the evolution of the objectives functions (E/E0) for the two optimization results (case 1 and 2). We note that with the use of the ECD the objective function decrease of about $80 \%$ at the first iteration. At the second and third iteration, the objective function decreases gradually until obtaining a profit of homogeneity of $93.5 \%$ compared to the initial exit velocities distribution.

With the use of the $\mathrm{CCD}$, the solution (total relative variation) obtained at the first iteration is higher than the initial solution. This is due to the weak evaluation points for the region of interest, which makes the approximation less accurate. On the other hand, at the second iteration, the objective function decreases by $85 \%$, then of $92.4 \%$ with the third iteration. The strategy of auto-adaptive search space makes it possible to enrich the approximation and to make it more accurate when we approach the global optimum.

The Fig. 9 represents the relative variation of the exit velocities distribution obtained by the initial die wall temperature and after optimization for both results (using two design of experiment). Qualitatively it is clear that the velocities distribution obtained with the initial temperature $\left(\mathrm{T}=230^{\circ} \mathrm{C}\right)$ gives more important relative variations, about $25 \%$ in the middle of the die and $50 \%$ at the edge. This important relative variation, result in lower velocities in the middle of the die, and more important velocities distribution on the edge. After optimization, we note that the velocities distribution is homogeneous over all the width of the die exit. The relative variations are lower than $5 \%$ over all the width of the die.

The optimal solution obtained for the two optimizations result (Fig. 10b and c), represents hotter temperatures of regulation in the middle of the die. The local flows are
Fig. 14 Distribution profile of optimal regulation temperature (ECD)

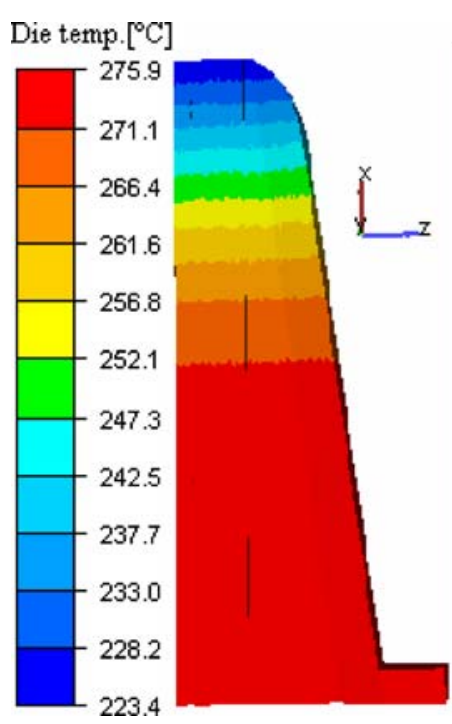


Fig. 15 Distribution profile of optimal regulation temperature (CCD)

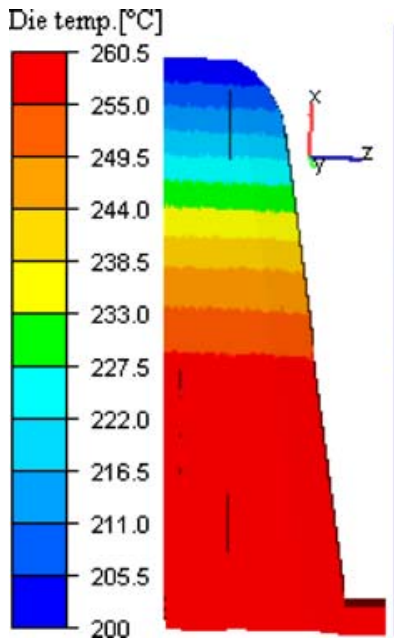

more important there and a compensation of the flow diminution is thus obtained compared to the initial regulation. On the other hand, the temperatures are lower on the edge to increase the polymer viscosity. This generates a reduction in the flow towards the edge. With these optimal temperatures of regulation, we could obtain a balance which makes it possible to give a homogeneous exit velocities distribution according to all the width on the die (Fig. 10b and c).

The Fig. 11 shows that the constraint on the pressure is respected for the two results (ECD and CCD). This constraint represents the ratio the pressure, in each iteration, and the pressure obtained with the initial temperature of regulation $\left(P^{e} / P_{0}^{e}\right)$. The optimization problem was formulated so that the ratio $\left(P^{e} / P_{0}^{e}\right)$ remains lower than one for each iteration, which gives a pressure decrease. We notes according to the constraints evolution according to the optimization iterations, that we obtains a profit of pressure of about $27 \%$ with the 3 rd iteration for the ECD (case 1 ). On the other hand, the pressure profit obtained by using a CCD (case2) is lower, it accounts for $15 \%$ compared to the initial pressure.

The pressure drop in the initial wall die temperature of regulation was specified as the limit pressure drop in the optimization algorithm. The goal was to improve the uniformity of exit velocity without increasing the pressure drop in the die. We show in the Fig. 12, that the pressure drop in the coathanger die is lower for the two results $(\mathrm{B}$, and $\mathrm{C})$. The optimal temperatures of regulation obtained by using the EDC (A) are higher. This made decrease the viscosity of polymer and thus implies a weaker pressure drop (see the study of the effect of the optimization variables on the pressure Fig. 5). The pressure is decreased by $27 \%$, compared to the limit pressure drop obtained by initial temperature of regulation. On the other hand, the temperatures of regulation obtained by using CCD (C) are lower compared to the result (B). This decrease the pressure drop by $16 \%$.

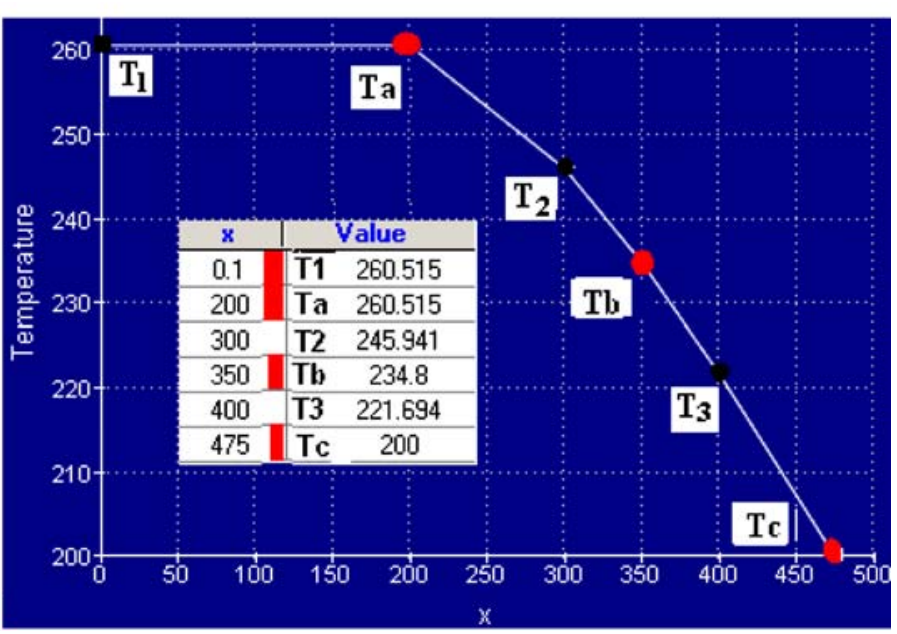

Figure 13 represents the evolution of the optimization variables (temperatures of regulation) during iterations of optimization. The solutions of the two results are different; on the other hand they have the same gradient. It is noted that the wall temperatures of regulation obtained by the first optimization (case1) are higher. The temperature in the middle of the die $\left(\mathrm{T}_{\mathrm{a}}\right)$ is about $276^{\circ} \mathrm{C}$, then it decrease according to the width of the die as illustrated on the Fig. 14 until the lowest temperature at the edge of the die $\mathrm{Tc}$, which is equal to $223.5^{\circ} \mathrm{C}$. It is noted that the difference of temperatures between $\ll T a, T b »$ and $\langle T b, T c »$, is respectively $19^{\circ} \mathrm{C}$ and $33^{\circ} \mathrm{C}$.

For Case 2, we notice that the temperatures are lower (Fig. 15). On the other hand, the differences in temperature between the various zones are a little stronger. These differences represent $25^{\circ} \mathrm{C}$ and $35^{\circ} \mathrm{C}$ respectively between the temperatures " $\mathrm{T}_{\mathrm{a}} \mathrm{T}_{\mathrm{b}}$ " and between " $\mathrm{T}_{\mathrm{b}} \mathrm{T}_{\mathrm{c}}$ " (see Table 3 ).

The Fig. 16 illustrates the Temperature distributions in the mid-plane of the initial (a), and optimized (b,c) temperature of regulation of the flat die. It is noted that

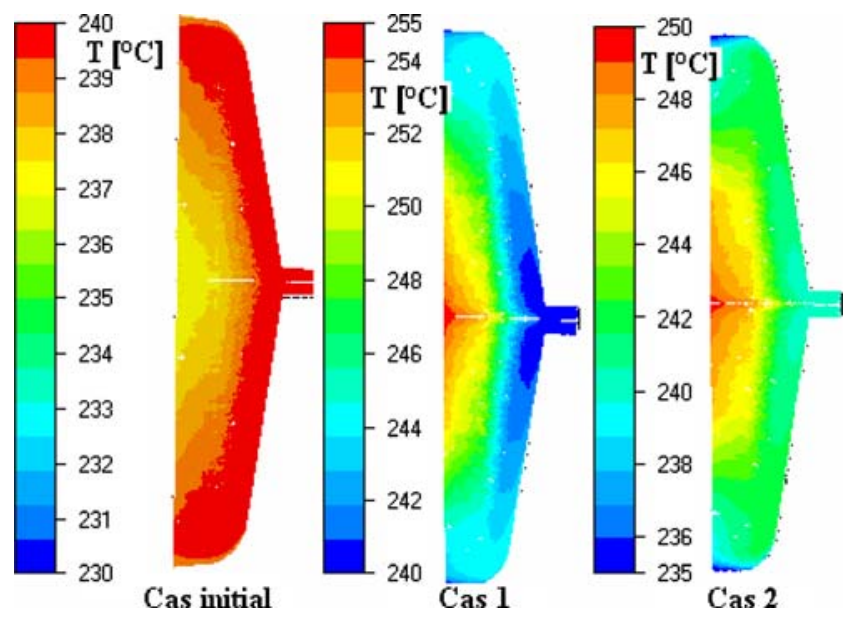

Fig. 16 Temperature distributions in the mid-plane of the initial (a), and optimized $(\mathbf{b}, \mathbf{c})$ temperature of regulation of the flat die 
for the temperature of initial regulation (constant wall die temperature), the melt temperature is higher on the edge and less low in the middle of the die. This difference in temperature which remains low $\left(\Delta \mathrm{T}_{\text {initials }}=3^{\circ} \mathrm{C}\right)$, be essential caused by viscous dissipation. However, we note that the difference in melt temperature obtained by the optimal wall die temperatures of regulation is more important according to the width of the die, because of the imposed temperatures of regulation. The difference in temperature is of $10^{\circ} \mathrm{C}$ for two optimizations $\left(\Delta T_{B} \approx \Delta T_{C} \approx 10^{\circ} \mathrm{C}\right)$. This difference in temperature can generate difficulties during drawing and cooling on the outlet side of the die.

\section{Conclusion}

In this paper, a procedure was applied in order to optimize the regulation temperatures distribution in a coat hanger die. The optimisation method is based on a Response Surface Method and has a very fast convergence, which is an advantage when time-consuming flow analysis calculations are involved.

The advantage of such an approach is its simplicity of implementation compared to geometrical optimization. A curve of control was introduced in order to decrease the number of optimization variables to 3 , while keeping 6 regulation temperatures. Two optimization cases were carried out according to the used experimental design. The two results show an improvement of the velocity distribution higher than $92 \%$ compared to the reference solution. The consideration of the nonlinear constraint allowed decreasing the pressure at the optimal design. Nevertheless, the weakness lies in the difference of the temperatures on the outlet side of the die. This is likely to generate difficulties during the operations of drawing and cooling. In the prospect we will optimize the geometry of a cylindrical die and will carry out experimental validations.

\section{References}

1. Nóbrega JM, Carneiro OS, Pinho FT, Oliveira PJ (2004) Flow balancing in extrusion dies for thermoplastic profiles. Int Polym Process 19:225-235

2. Ettinger HJ, Sienz J, Pittman JFT, Polynkin A (2004) Parameterization and optimisation strategies for the automated design of U PVC profile extrusion dies. Struct Multidisc Optim 28: 180-194

3. Michaeli W, Kaul S, Wolff T (2001) Computer aided optimisation of extrusion dies. J Polym Eng 21:225-237

4. Lebaal N, Schmidt F, Puissant S, Schlafli D (2009) Design of optimal extrusion die for a range of different materials. Polym Eng Science 49:432-440

5. Smith DE (2003) An optimisation-based approach to compute sheeting die designs for multiple operating conditions. SPE ANTEC Tech. Papers, 315-319

6. Wang Y (1991) The flow distribution of molten polymers in slit dies and coat hanger die through three-dimensional finite element analysis. Polym Eng Sci 31:204-212

7. Vergnes B, Saillard P, Agassant JF (1984) Non-isothermal flow of a molten polymer in a coat-hanger die. Polym Eng Science 24:980-987

8. Schläfli D (1995) Analysis of polymer flow through coat-hanger melt distributors. Int Polym Process 10:195

9. Vergnes B, Agassant JF (1995) Modélisation des écoulements dans les filières d'extrusion. Techniques de l'Ingénieur, traité Plastiques et Composites, A3 655:1-19

10. Fradette L, Tanguy PA, Thibault F, Sheehy P, Blouin D, Hurez P (1995) Optimal-design in profile extrusion calibration. J Polym Eng 14:295-322

11. Myers RH, Montgomery DC (2002) Response surface methodology, process and product optimization using designed experiments, second editionth edn. Wiley interscience publication, USA

12. Montgomery DC (2005) Design and analysis of experiments. John wiley \& Sons, INC, USA

13. Lebaal N, Puissant S, Schmidt FM (2005) Rheological parameters identification using in-situ experimental data of a flat die extrusion. J Materials Process Tech 164:1524-1529

14. Kaymaz I (2005) Application of Kriging method to structural reliability problems. Struct Safety 27:133-151

15. Lebaal N, Schmidt F, Puissant S (2009) Design and optimization of three dimensional extrusion dies, using constraint optimization algorithm. Finite Elem Anal Des 45:333-340 